\title{
ATLAS Jet Trigger Update for the LHC Run II
}

\section{Ademar Delgado}

On behalf of the ATLAS Collaboration

LIP - Lisbon

ANIMMA 2015

20-24 April 2015
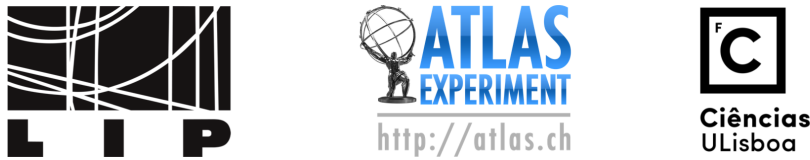


\section{Introduction: The Large Hadron Collider (LHC) at CERN}

- Circular accelerator with $27 \mathrm{~km}$ perimeter

- Proton-proton collider

- Can also collide heavy ions
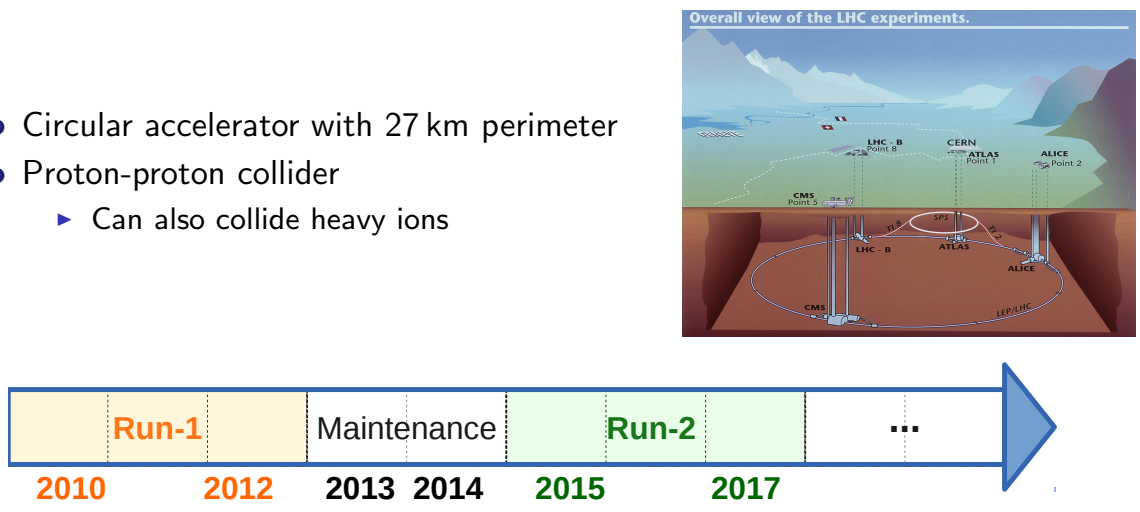

Centre of mass energy Bunch spacing Interactions per bunch crossing Peak luminosity

\begin{tabular}{c|c} 
During Run-1 & For Run-2 \\
\hline $7 \mathrm{TeV}$ to $8 \mathrm{TeV}$ & $13 \mathrm{TeV}$ to $14 \mathrm{TeV}$ \\
$50 \mathrm{~ns}$ & $25 \mathrm{~ns}$ \\
20 & $\sim 43$ \\
$0.7 \times 10^{34} \mathrm{~cm}^{-2} \mathrm{~s}^{-1}$ & $1.6 \times 10^{34} \mathrm{~cm}^{-2} \mathrm{~s}^{-1}$ \\
& ATL-DAQ-PROC-2014-032
\end{tabular}




\section{Introduction: The ATLAS experiment}

Multi-purpose experiment designed to probe the Standard Model and search for new physics at very high energies

Muon Spectrometer: $|\eta|<2.7$

Air-core toroids and gas-based muon chambers

o/pT=2\%@50GeV to 10\%@1TeV (ID+MS)

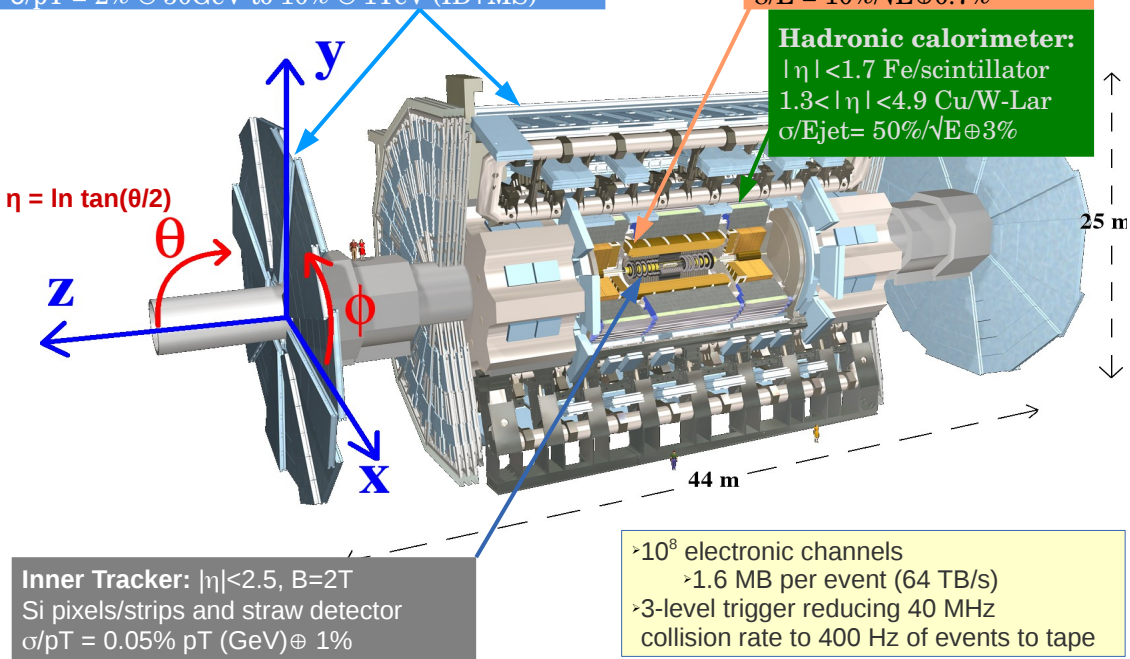




\section{Jet: Spray of collimated particles}

- Initiated by quarks or gluons

- Most common high $p_{T}$ objects produced at the LHC

- Important for a wide range of physics analyses

- The energy deposits are aggregated by the reconstruction algorithms and calibrated to provide the jet momentum measurement

\section{$\otimes$ ATLAS

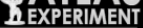

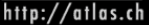
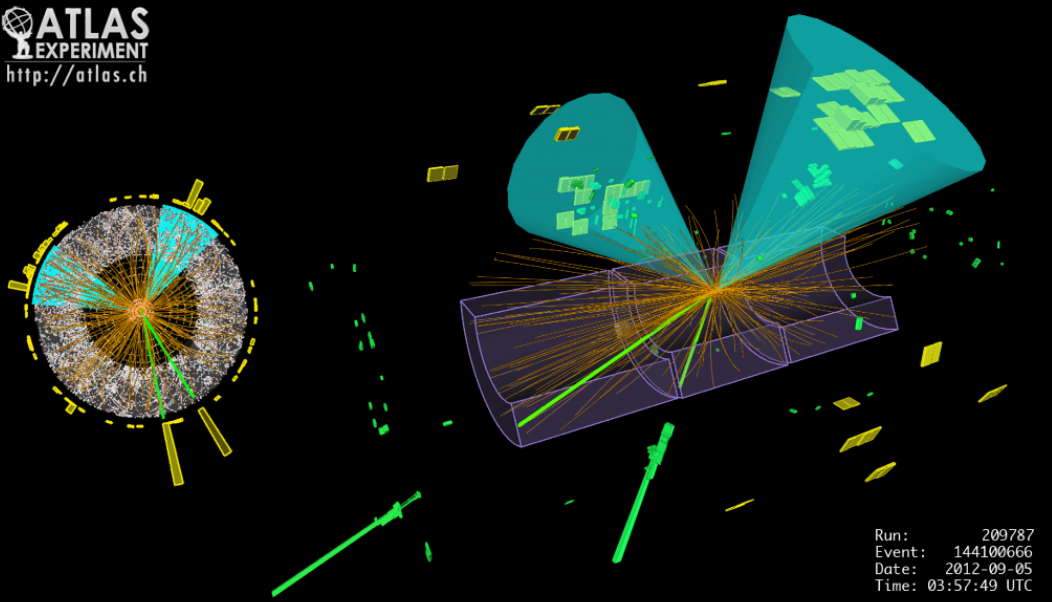


\section{The ATLAS Trigger and DAQ systems: Run-1}

- Reduce the accepted events by a factor of $10^{5}$

DOI:10.1140/epjc/s10052-011-1849-1

- Most interesting physics have small cross-section relative to total p-p cross-section

- Limited bandwidth to disk data storage

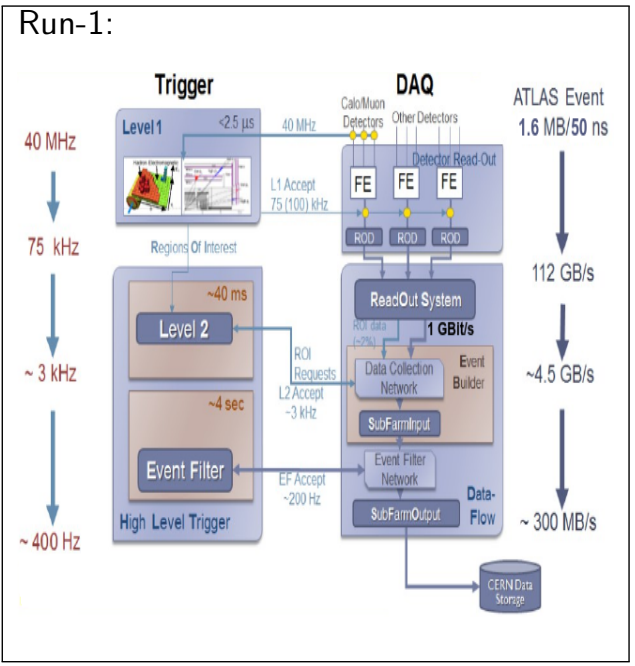

- Organized in 3 levels:

- L1: Hardware based

$\star$ Coarse granularity.

* Finds high $p_{T}$ objects positions

$\star$ Provides seeds for next levels

- L2: Software based

$\star$ Full granularity

$\star$ Regions of interest based

- EF: Software based

$\star$ Offline like algorithms

$\star$ Can access the full event

- L2 and EF in different farms

- Extra data transfer 


\section{The ATLAS Trigger and DAQ systems: Run-1}

- Reduce the accepted events by a factor of $10^{5}$

DOI:10.1140/epjc/s10052-011-1849-1

- Most interesting physics have small cross-section relative to total p-p cross-section

- Limited bandwidth to disk data storage

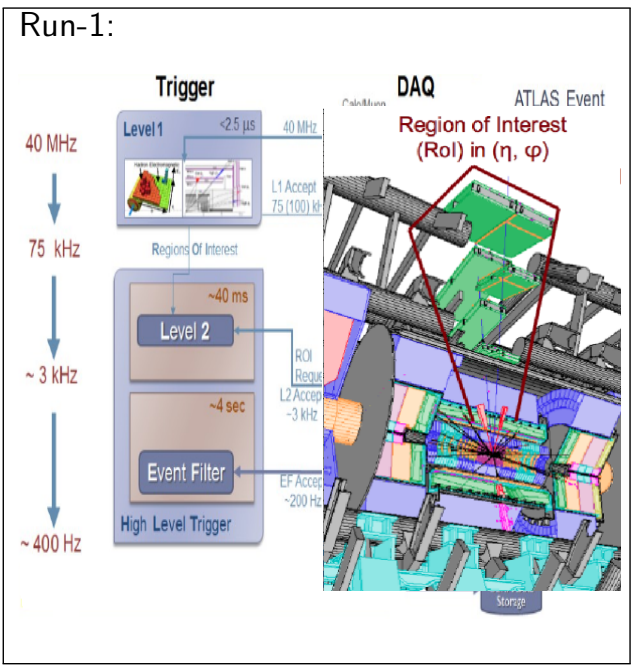

- Organized in 3 levels:

- L1: Hardware based

$\star$ Coarse granularity.

$\star$ Finds high $p_{T}$ objects positions

$\star$ Provides seeds for next levels

- L2: Software based

$\star$ Full granularity

$\star$ Regions of interest based

- EF: Software based

$\star$ Offline like algorithms

$\star$ Can access the full event

- L2 and EF in different farms

- Extra data transfer 


\section{The ATLAS Trigger and DAQ systems: Run-1}

- Reduce the accepted events by a factor of $10^{5}$

DOI:10.1140/epjc/s10052-011-1849-1

- Most interesting physics have small cross-section relative to total p-p cross-section

- Limited bandwidth to disk data storage

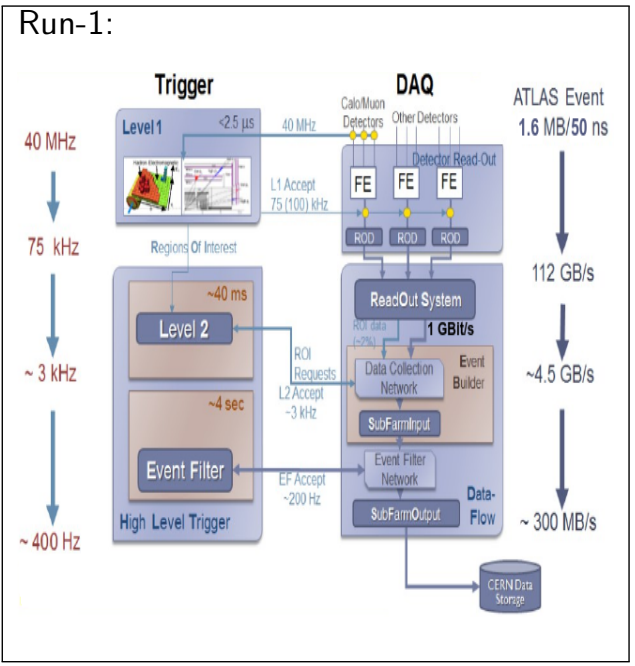

- Organized in 3 levels:

- L1: Hardware based

$\star$ Coarse granularity.

* Finds high $p_{T}$ objects positions

$\star$ Provides seeds for next levels

- L2: Software based

$\star$ Full granularity

$\star$ Regions of interest based

- EF: Software based

$\star$ Offline like algorithms

$\star$ Can access the full event

- L2 and EF in different farms

- Extra data transfer 


\section{The ATLAS Trigger and DAQ systems: Run-2}

- Improve the trigger capability for Run-2

- Better exploit available computing capacity and output rate from the HLT

- New L1 topological processor and fast tracking at HLT:

$\star$ will allow angular and mass jet selection and rejection of pileup jets

- Improved data flow architecture

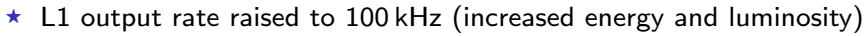

$\star$ L2 and EF merged; 250 ms to take a decision for jets

$\star$ Read Out System upgrade; bandwidth increase 10x, memory per channel $5 x$
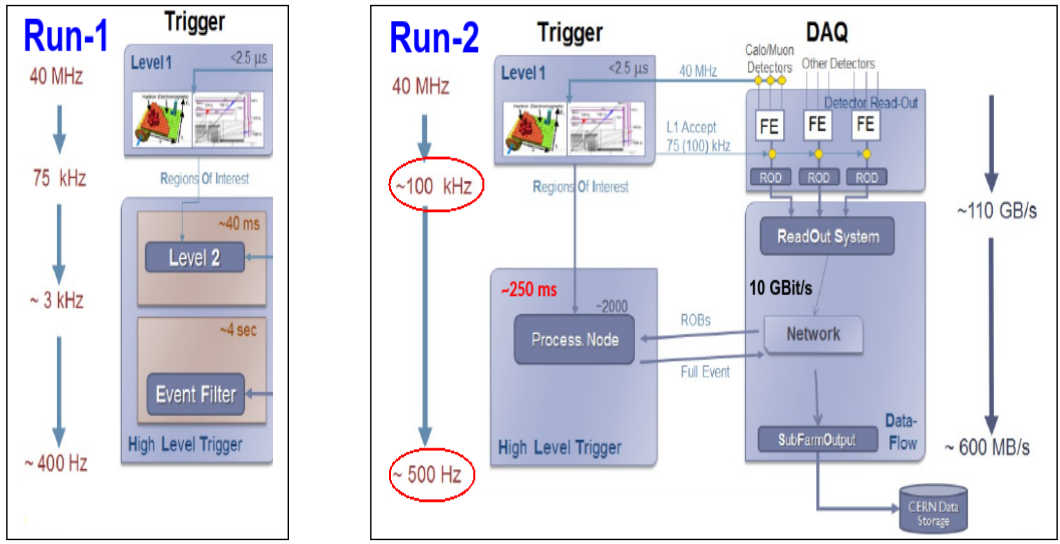


\section{Jet trigger: Run-1}

- Selects events containing high $p_{T}$ hadronic jets Typical chain for single Jet trigger

- L1:

- Sliding window algorithm

- Calorimeter towers (trigger towers)

- L2:

- Readout Regions of Interest

- Simple cone algorithm

- Cells as constituents

- EF:

- Full calorimeter scan

- Offline algorithms available
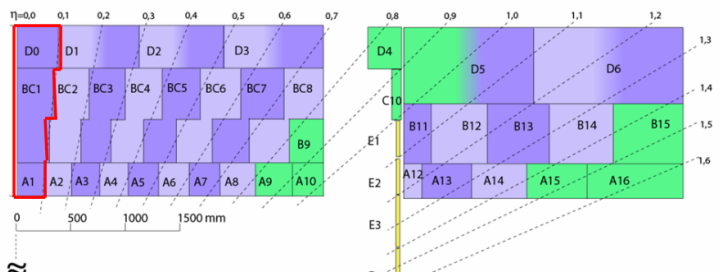

Run-1

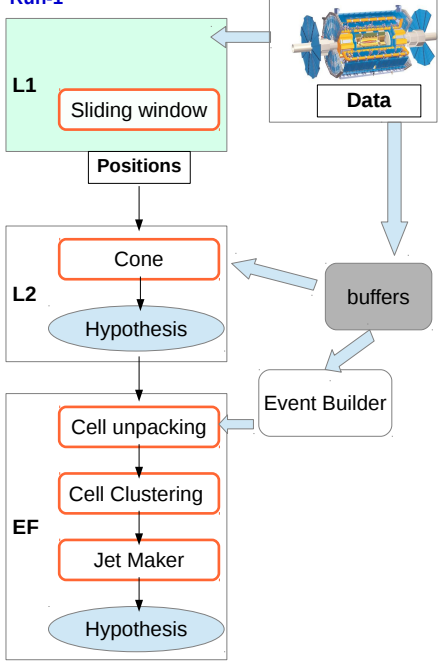




\section{Jet trigger: Run-2}

- Selects events containing high $p_{T}$ hadronic jets Typical chain for single Jet trigger

- L1:

- Sliding window algorithm

- Calorimeter towers (trigger towers)

- HLT generic jet chain:

- Prepare detector data (Cell unpacking)

- Process cell Clustering and Jet maker

- Evaluate the event (Hypothesis)

Topological Clustering algorithm:

- Iterative 3D calorimeter cell clustering

- based on signal/noise

- Applies noise suppression

- Most time-demanding step

Jet reconstruction uses $A n t i-k_{\perp}$ algorithm [1]

- Fast, collinear and infrared safe and regular shape algorithm

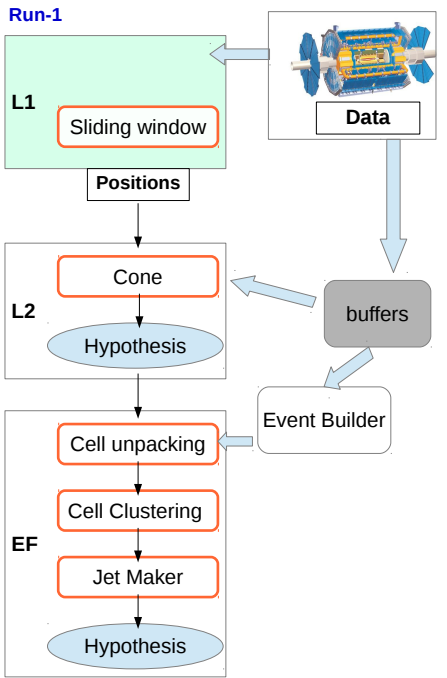

[1] JHEP 0804:063,2008 


\section{Jet trigger: Run-2}

- Selects events containing high $p_{T}$ hadronic jets

Typical chain for single Jet trigger

- L1:

- Sliding window algorithm

- Calorimeter towers (trigger towers)

- HLT generic jet chain:

- Prepare detector data (Cell unpacking)

- Process cell Clustering and Jet maker

- Evaluate the event (Hypothesis)

Topological Clustering algorithm:

- Iterative 3D calorimeter cell clustering

- based on signal/noise

- Applies noise suppression

- Most time-demanding step

Jet reconstruction uses $A n t i-k_{\perp}$ algorithm [1]

- Fast, collinear and infrared safe and regular shape algorithm

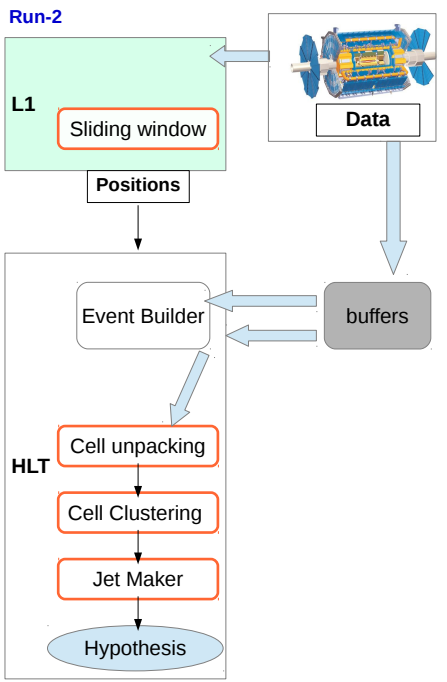

[1] JHEP 0804:063,2008 


\section{Jet trigger: Run-2}

- Selects events containing high $p_{T}$ hadronic jets

Typical chain for single Jet trigger

- L1:

- Sliding window algorithm

- Calorimeter towers (trigger towers)

- HLT generic jet chain:

- Prepare detector data (Cell unpacking)

- Process cell Clustering and Jet maker

- Evaluate the event (Hypothesis)

- Jet event rate increase by a factor of five

- higher energy, luminosity and pileup

- Better exploit available computing capacity

- HLT algorithms optimization

- Data reduction through the use of a partial calorimeter readout scheme

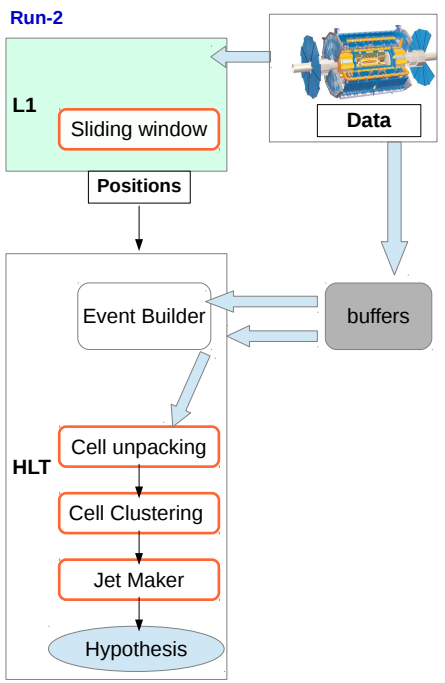




\section{Algorithm optimization}

- The cell unpack and retrieval time was reduced by a factor of 7

- More efficient full calorimeter readout

- The cell clustering time was reduced by a factor of 2

- Pre-fetching, code inlining and the introduction of more efficient data structures

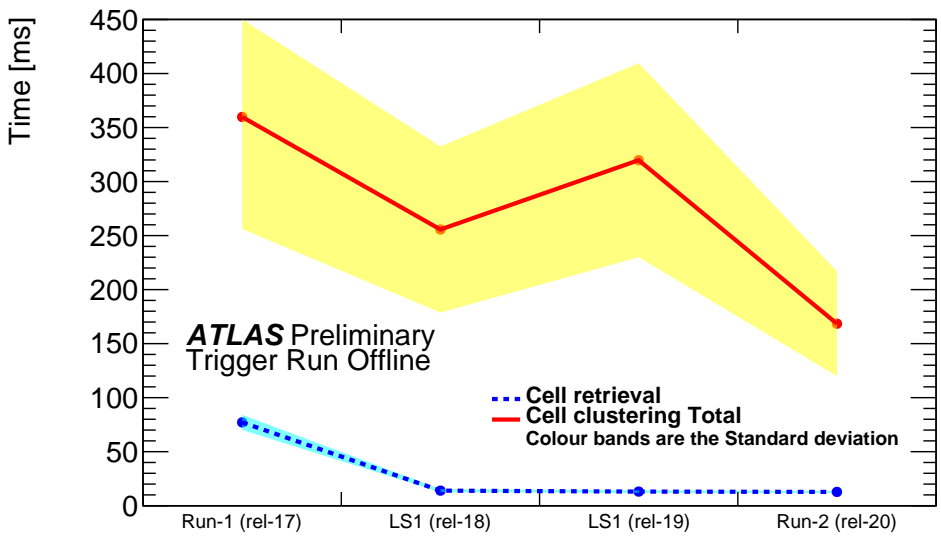

Software release 


\section{Data reduction: Partial calorimeter readout scheme}

- Reads at once all the cells around the L1 positions, removing any overlap

- Works as a full calorimeter readout with low activity regions suppression

- zero-suppressing algorithm

- Increases system flexibility to adapt to different requirements

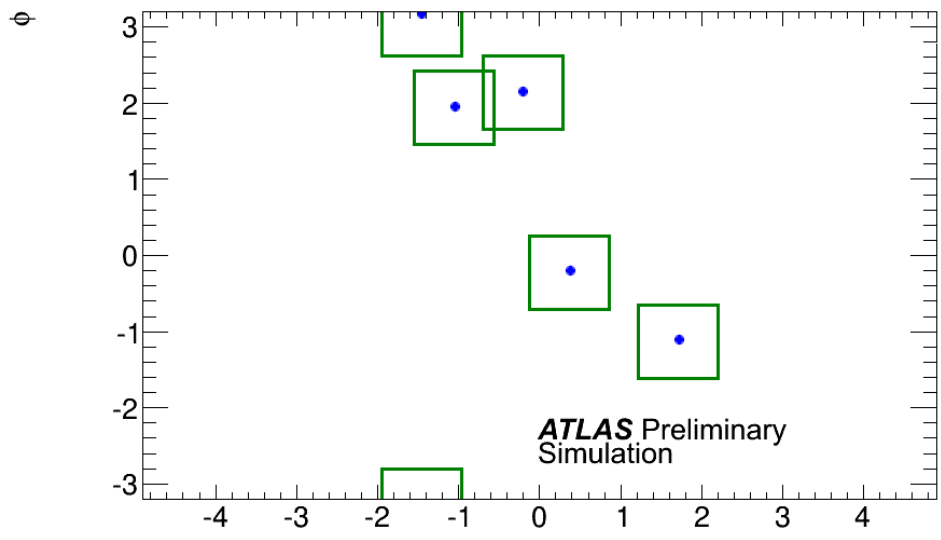

$\eta$ 


\section{Partial Scan: Data and processing time reduction}

- The Partial Scan reads out 3.5 to $7 \%$ of the calorimeter cells

- The cell clustering in the Partial Scan takes 6 to $10 \%$ of the time required by the complete calorimeter processing
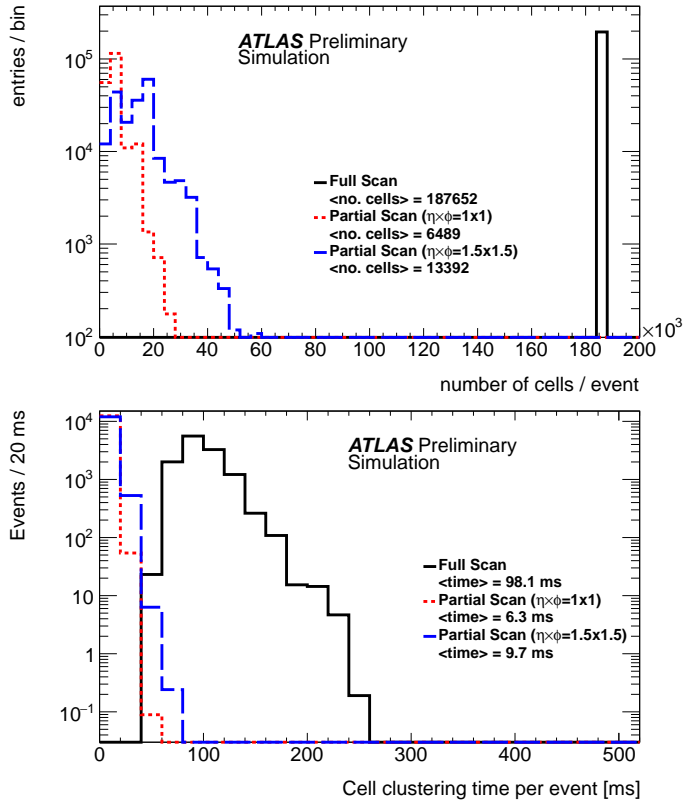


\section{Partial Scan: Comparing partial to full reconstruction}

- The full calorimeter readout gets the best resolution

- The $E_{T}$ of the most central jet differs by less than $0.5 \%$ from full scan trigger jets, for jets above $100 \mathrm{GeV}$

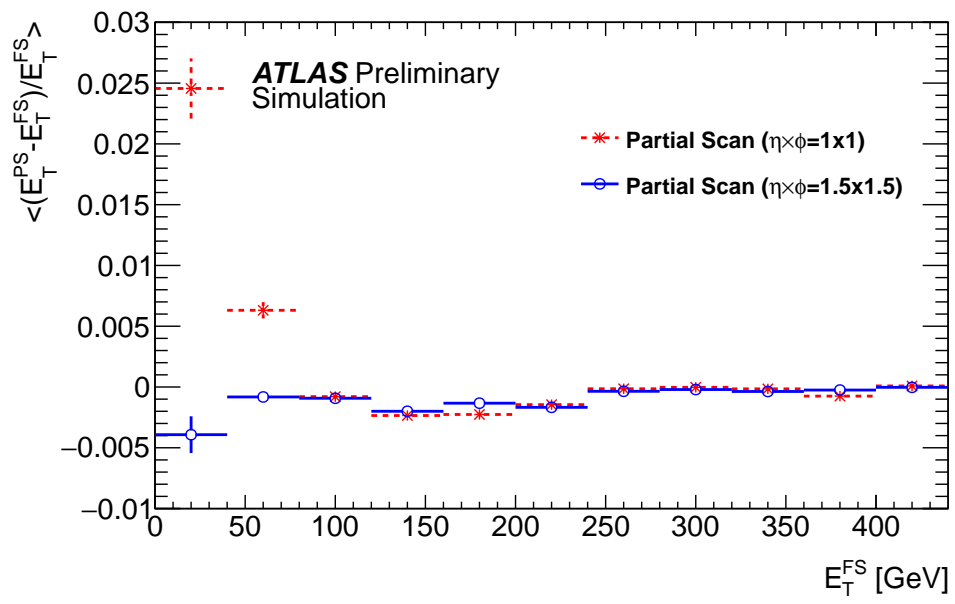




\section{Conclusions:}

- The jet trigger has shown excellent performance during the first data taking

- The ATLAS TDAQ introduced important improvements to better exploit Run-2

- Significant optimizations were achieved during shut-down:

$\star$ cell unpacking becomes seven times faster

$\star$ cell clustering processing time was reduced by a factor of two

- A partial calorimeter readout scheme was developed:

* zero-suppressing algorithm that filters the low activity regions

$\star$ Speeded up jet reconstruction by a factor of 10 without much loss of physics performance

- The ATLAS jet trigger will collect crucial data to calibrate the detector and allow many unique physics measurements. We are ready to face the challenges of the LHC Run-2! 


\section{Thank you!}

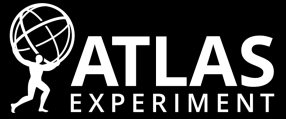

Run: 260272 Event: 5879 2015-04-05 10:46:41 CEST

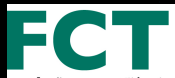

Fundação para a Ciência e a Tecnologia

\section{Ackribyledgements}
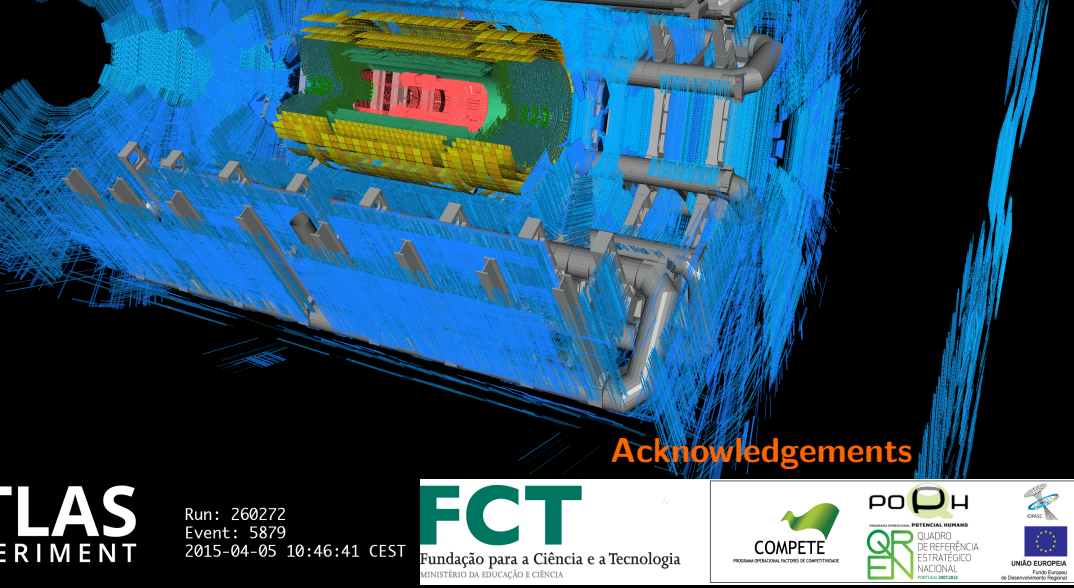


\section{Backup slide: Topological Cluster}
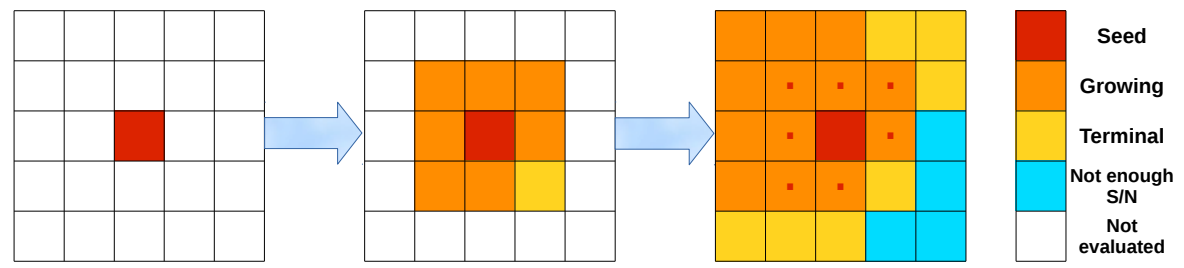